\title{
Influence of Ceiling Height on Airflow and Particle Distribution in an Operating Room
}

\author{
Aleyna Agirman ${ }^{1, *}$, Yunus Emre Cetin ${ }^{2}$, Mete Avci ${ }^{1}$ and Orhan Aydin ${ }^{1}$ \\ ${ }^{1}$ Department of Mechanical Engineering, Karadeniz Technical University, 61080 Trabzon, Turkey \\ ${ }^{2}$ Arsin Vocational School, Karadeniz Technical University, 61910 Trabzon, Turkey
}

\begin{abstract}
Bacteria-carrying particles released by surgical staff are the main factors leading to surgical site infections (SSI). Operating rooms must be designed to reduce risks of such infections. In this numerical study, the effect of different ceiling heights on particle dispersion and deposition are studied for an operating room with laminar airflow (LAF) system. Two different particle diameters $(12$ and $20 \mu \mathrm{m})$ are tested under three different ceiling heights $(2.43-2.8-3.0$ $\mathrm{m})$ at a constant air change rate $(\mathrm{ACH}=20)$. Numerical predictions are performed by using the commercial software ANSYS Fluent 16.0. The results reveal that decreasing of the ceiling height reduces the amount of particle deposited on the surgical table.
\end{abstract}

\section{INTRODUCTION}

It is very important to provide sustainable hygienic environment along with clinical needs for operating rooms (OR). Surgical staff is the main source of bacteria carrying particles (BCPs) in an operating room. Particle size considered as infection risk at the surgical staff and patient is from $4 \mu \mathrm{m}$ to $20 \mu \mathrm{m}$ [1]. During a surgery, most surgical staff is located near the surgical table, which increase the possible transmission of BCPs to the open tissue. The amount of released particles from the staff depends on many factors like clothing, posture and activity level. Mean value of particles released from a surgical staff is $1.5 \mathrm{BCP} / \mathrm{s}$ [2].

A LAF unit is expected to minimize particle concentration on the surgical site and reduce the infection risk by creating a unidirectional flow over the surgical table. The factors that can effect this flow and diminish the washing effect created by LAF need to be well understood. For a better dilution and/or removal of particles ventilation system [3-5], inlet/outlet positions [69] and air change rate [10 and11] should be analyzed. To the authors' best knowledge, there is only one study [12] dealing with the effect of ceiling height on particle dispersion on the surgical site. In this regard, the aim of this study is to investigate effect of the ceiling height on particle distribution and deposition in an operating room with LAF via computational fluid dynamics.

\section{NUMERICAL METHOD}

\subsection{Geometry}

Figure 1 shows the physical arrangement of the operating room examined, which is in identical size of located in Karadeniz Technical University Hospital (Trabzon, Turkey). The dimensions of OR are $6.5 \mathrm{~m}$ in length and $9.43 \mathrm{~m}$ in width $(\mathrm{W})$ with a floor-to-ceiling height of 2.43 $\mathrm{m}(\mathrm{H})$. The supply air diffuser covers a projected area of $1.8 \mathrm{~m}(\mathrm{~L}) \times 2.4 \mathrm{~m}(\mathrm{~W})$. The operating room contains a medical lamp, two instrument tables, medical equipment, a surgical table, four surgical staff $(1.75 \mathrm{~m})$ and four airexhaust outlets at each corner. Three different ceiling heights $(2.43 \mathrm{~m}, 2.8 \mathrm{~m}$ and $3.0 \mathrm{~m})$ are considered for a constant air change rate $(20 \mathrm{ACH})$. Particles with a diameter of $12 \mu \mathrm{m}$ and $20 \mu \mathrm{m}$ at a density of $1000 \mathrm{~kg} / \mathrm{m}^{3}$ are released from the mouth of surgical team members.

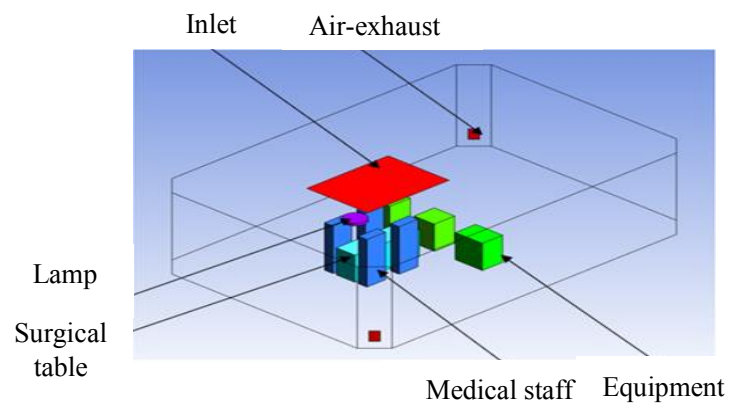

Figure1.Geometric configuration of the operating room

${ }^{*}$ Corresponding author: aleynaagirman $@$ ktu.edu.tr 


\subsection{Numerical Model}

\subsubsection{Airflow Simulation Model}

The Reynolds-averaged Navier-Stokes equations are solved numerically by finite volume method with the well-known realizable $k-\varepsilon$ model to describe the flow field. The governing equations for continuity, momentum and energy may be expressed in the general form as follows:

$$
\frac{\partial(\rho \phi)}{\partial t}+\Delta \cdot(\rho \phi \vec{V})=\Delta \cdot\left(\Gamma_{\phi} \Delta_{\phi}\right)+S_{\phi}
$$

where $\vec{V}$ is the air velocity vector, $\rho$ is the air density, $\phi$ is the transported quantity, $\Gamma_{\phi}$ is the diffusion coefficient of $\phi$ and $S_{\phi}$ is the source term.

Velocity inlet and outflow boundary conditions are used for inlet and outlet, respectively. Adiabatic boundary conditions and non-slip velocity are set at all solid wall boundaries. The air temperature is defined as $20{ }^{\circ} \mathrm{C}$ and turbulence intensity is set as $10 \%$. The discretization scheme used for all variables is the second-order upwind scheme. The SIMPLE algorithm is adopted to couple velocity and pressure. Convergence criteria for all equations are set to $1 \times 10^{-5}$. Enhanced wall treatment is employed to treat the turbulent flow properties in the nearwall regions. Because of the complex geometry, an average of 6.5 million hexahedral cells are used to carry out the airflow simulation. A grid refinement study is performed using three different grids, resulting in $1 \leq$ $y^{+} \leq 7$ for the entire computational domain. The commercial software FLUENT 16 is used to perform all the simulations.

\subsubsection{Numerical Model of Particle Motion}

Two approaches are used to simulate airborne particles dispersion: the Eularian and Lagrangian particle tracking models. Here, the particle trajectories are tracked using the Lagrangian approach. In this approach, each particle trajectory is calculated by integrating the force balance equation:

$$
\frac{d u_{p}}{d t}=F_{D}\left(u-u_{p}\right)+\frac{g\left(\rho_{p}-\rho\right)}{\rho_{p}}+F_{e}
$$

where $\rho_{p}$ and $\rho$ are particle and air density, $u$ and $u_{p}$ are the velocity vector of the air and particle, respectively and $g$ is the gravitational acceleration. The first part on the right side of the equation represents the drag force while the left side represents the inertia force. The term $F_{e}$ is the additional force (Brownian force, Thermophoretic force, Saffman's lift force). $F_{D}$ is the inverse of the relaxation time $\left(\mathrm{s}^{-1}\right)$ and is given by:

$$
F_{D}=\frac{18 \mu}{\rho_{p} d_{p}^{2}} \frac{C_{d} \operatorname{Re}_{p}}{24}
$$

where $\mu$ is the molecular viscosity of air and $d_{p}$ is the particle diameter. $C_{d}$ is the drag coefficient which is defined as:

$$
C_{d}=\frac{\xi_{1}}{\operatorname{Re}_{p}}+\frac{\xi_{2}}{\operatorname{Re}_{p}^{2}}+\xi_{3}
$$

where $\zeta_{1}, \zeta_{2}$, and $\zeta_{3}$ are constants given by Morsi et al. [13]. $R e_{p}$ is the relative Reynolds number based on the particle diameter:

$$
\operatorname{Re}_{p}=\frac{\rho d_{p}\left|u_{p}-u\right|}{\mu}
$$

Particle dispersion due to turbulence is calculated by the discrete random walk (DRW) model. Because of the stochastic nature of the DRW model, a sufficient number of trajectories should be calculated to obtain statistically reliable solution [13]. It is found that concentration results became statistically stable at about $10^{6}$ trajectories. After the turbulent airflow area is analyzed, particles are injected into the room. Bacteria-carrying particles with an aerodynamic diameter of $12 \mu \mathrm{m}$ and $20 \mu \mathrm{m}$ are released from the mouth of surgical staff to the operating room air. Escape boundary conditions are set for the particles leaving the OR via outlets, and trap boundary conditions are considered for all other solid surfaces. This boundary condition implies that the particles that will come into contact with the surfaces will not be resuspended by air. No recycling from the walls of the particles is assumed.

\subsubsection{Verification of Mathematical Model}

The experimental results of Chen et al. [14] are employed to check the validity of the numerical model.

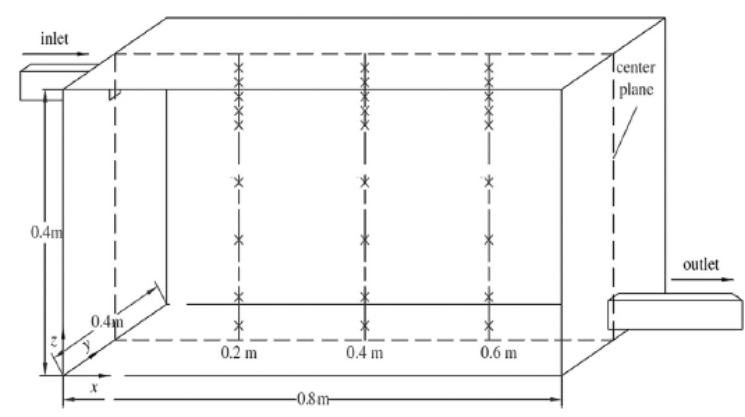

Figure 2. The geometry of the model room [14] 
The model room dimensions are $\mathrm{L} \times \mathrm{W} \times \mathrm{H}=0.8 \mathrm{~m} \times 0.4$ $\mathrm{m} \times 0.4 \mathrm{~m}$. The inlet and outlet vents are symmetrical in relation to the center plane and the dimensions are $0.04 \mathrm{~m}$ x $0.04 \mathrm{~m}$. Inlet velocity is $0.225 \mathrm{~m} / \mathrm{s}$. The particle size released into the chamber is $10 \mu \mathrm{m}$ and the material density is $1400 \mathrm{~kg} / \mathrm{m}^{3}$.
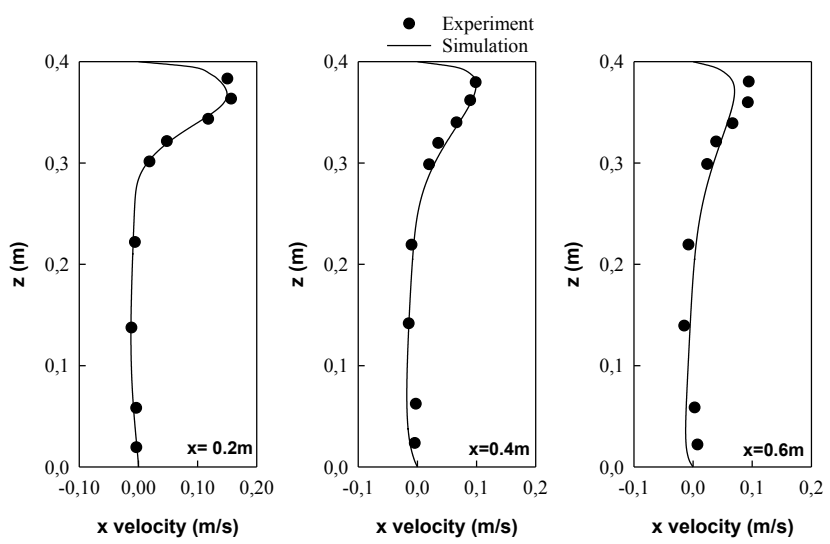

Figure 3: Comparison of measured [14] and predicted velocity at three different locations
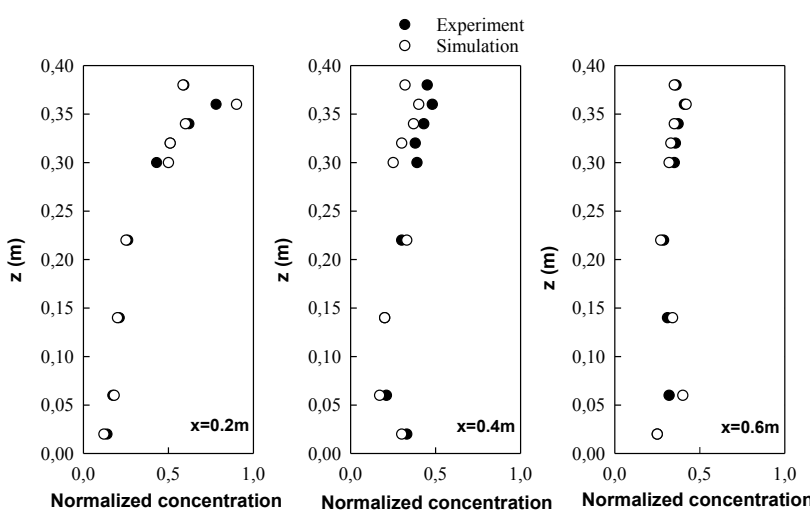

Figure 4: Comparison of measured [14] and predicted concentrations at three different locations

In Figures 3 and 4 , $\mathrm{x}$-velocity and concentration predictions are compared with experimental data [14]. Both velocity profile and Lagrangian DRW simulations are found to be compatible with measurements, which validates the numerical method.

\section{RESULTS AND DISCUSSION}

Figure 5 shows the velocity contours and streamlines in the center plane of the surgical table at three different ceiling heights. As it is seen from the figure, the magnitude of the air velocity over the surgical table decreases with an increase in the ceiling height. This decrease in the air velocity weakens the drag force which acts on the BCPs and leads more deposition on surgical table. It is also seen that the medical lamp located on the surgical table disrupts the unidirectional air flow pattern and forms a stagnant zone in the lower region of the lamp. The blockage of the unidirectional flow can lead more deposition of the BCPs to the surgical table from the surgeons inside this area.

a)

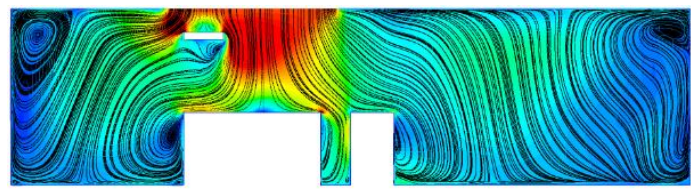

b)

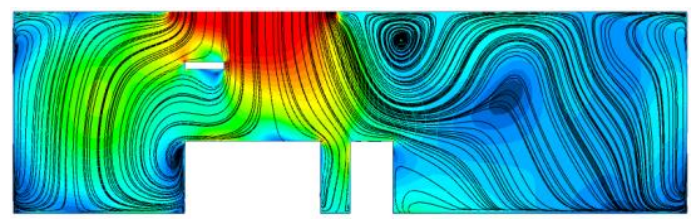

c)

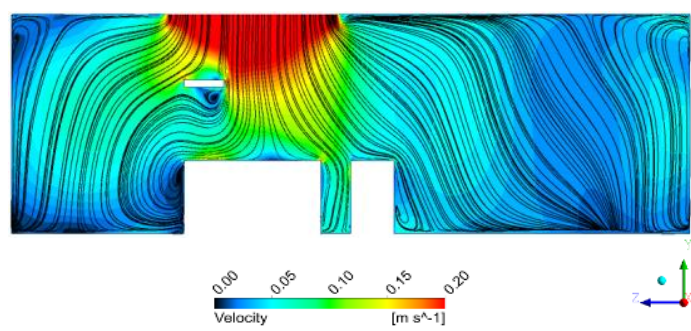

Figure 5: Velocity-contours and streamlines plot at the center-plane of the operating room (20 ACH); (a) $2.43 \mathrm{~m}$, b) $2.8 \mathrm{~m}$, (c) $3 \mathrm{~m}$

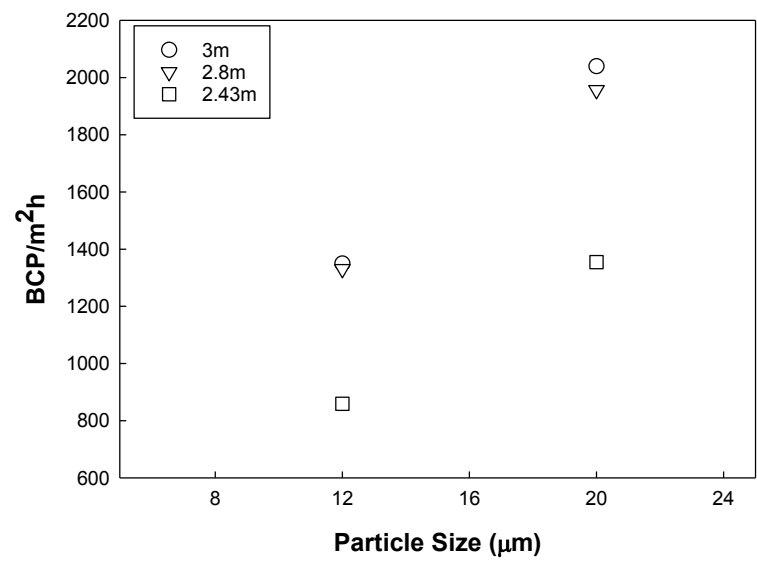

Figure 6: Amount of particle deposited on the surgical table at three different ceiling heights

Figure 6 shows the amount of particle deposited on the surgical table for three different values of the ceiling height and two different values of the particle diameter. It is seen that the amount of particles deposited on the surgical table reduces with a decrease in both the ceiling height and the particle diameter. This an expected result since a decrease in the ceiling length increases the rate of 
drag forces to gravitational forces which is the driving mechanism in sweeping the particles.

Figure 7 shows particle concentration contours of $20 \mu \mathrm{m}$ particles on the height of the surgical table $(\mathrm{y}=1 \mathrm{~m})$ for all the three heights considered. As it is seen in Fig. 7a, parallel down flow to the surgical table is strong enough to create a particle free area which is not achieved for other ceiling height conditions because of the weak airflow.
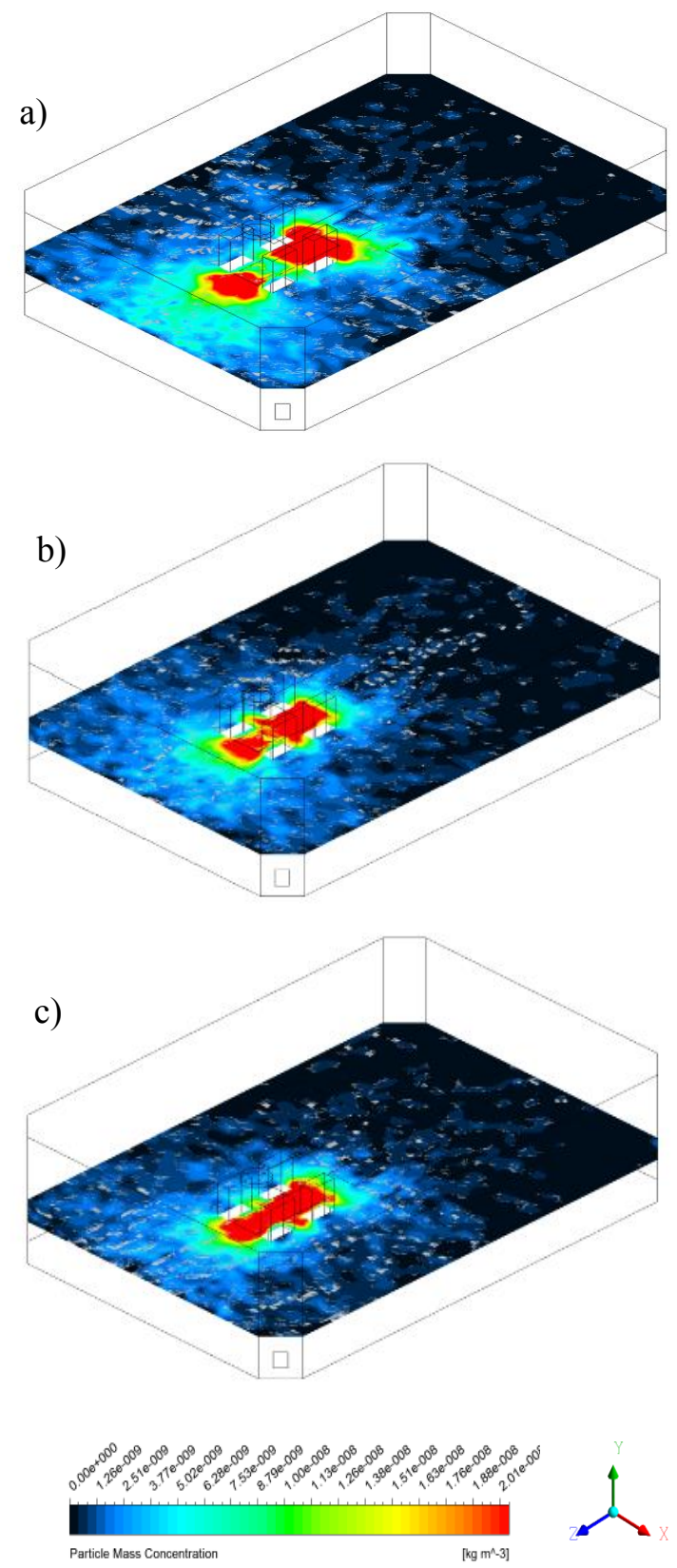

Figure 7: Particle concentration on the height of surgical table ( $\mathrm{y}=1 \mathrm{~m}$ ) for $20 \mu \mathrm{m}$. (a) $2.43 \mathrm{~m}$, (b) $2.8 \mathrm{~m}$, (c) $3 \mathrm{~m}$

\section{CONCLUSIONS}

In this paper, the Realizable $k-\varepsilon$ turbulence model and the Lagrangian approach are used to simulate the effect of ceiling height on airflow distribution and particle dispersion in the operating room, respectively. The results indicate that the particle concentration around the surgical site is highly correlated with the distance of LAF to the surgical table, and the number of particles impinge on the critical area decreases with a decrease in the ceiling height. For higher ceiling heights, higher air change rates should be evaluated.

\section{REFERENCES}

1. W.C. Noble, O.M. Lidwell, D. Kingston, J. Hyg. (Lond) 61,385-391 (1963).

2. S. Sadrizadeh, S. Holmberg, Proc. 13th SCANVAC Int. Conf. Air Distrib. Rooms (2014).

3. F. Memarzadeh, A. Manning, ASHRAE Transactions 108, 3-15 (2002).

4. C. Wang, S. Holmberg, S. Sadrizadeh, Building and Environment, 144, 45-56 (2018).

5. M. Alsved, A. Civilis, P. Ekolind, A. Tammelin, AE. Andersson, J. Jakobsson, T. Svensson, M. Ramstorp, S. Sadrizadeh, P.A. Larsson, M. Bohgard, T. SantlTemkiv, J. Löndahl, J. of Hosp. Infect. 98, 181-190 (2018).

6. S. Sadrizadeh, S. Holmberg, Proc. 35th AIVC, 4th Tightvent 2nd Vent. Conf. (2014).

7. T.N. Verma, A.K. Sahu, S.L. Sinha, Proceedings, 4,10074-10079 (2017).

8. R. Kumar, R. Kumar, A. Gupta, International Journal of Ventilation,7:2, 139-149 (2016).

9. M. Ning, S. Mengjie, C. Mingyin, P. Dongmei, D. Shiming, Applied Energy, 164,906-915 (2016).

10. S. Sadrizadeh, S. Holmberg, A. Tammelin, Building and Environment, 82, 517-525 (2014).

11. R. Al-Waked, Engineering Applications of Computational Fluid Mechanics, 4:1, 1-16 (2014).

12. F Memarzadeh, Z. Jiang, In: Proceedings of IAQ (2004).

13. S. A. Morsi, A.J. Alexander, Journal of Fluid Mechanics, 55(02), 193-208 (1972).

14. F. Chen, S.C.M. Yu, A.C.K. Lai, Atmospheric Environment, 40:357-367 (2006). 